\title{
Multicast Dual Arti-Q System in Vehicular Adhoc Networks
}

\author{
S.Sujatha ${ }^{1}$, P.Sukanya ${ }^{2}$ \\ ${ }^{1}$ (Associate Professor, Computer Science, Dr.G.R.D College of Science, India) \\ ${ }_{2}^{2}$ (Research Scholar, Computer Science, Dr.G.R.D College of Science, India)
}

\begin{abstract}
The rising requirements of the wireless communication increase the researches in the wireless communication area. Wireless communication is used in Ad hoc network. MANET is one of the self organizing, self healing network in the Ad hoc network. VANET is a subset of the MANET which offers console and protection to the users of VANET. VANET is a collected works of vehicular mobile nodes which figures the vehicular Ad hoc networks. In VANET there are many confronting wanted to solve, in order to provide efficient and stable services. Existing paper represents the Artigence techniques which will solve the challenges in the $V A N E T$ and improve the efficiency. Artigence uses Arti-Q algorithm, which is one type of efficient queuing technique. The Arti-Q and VANET are using in the call taxi management systems in order to provide efficient call taxi management service to the customers.
\end{abstract}

Keywords-Wireless Communication, Artigence techniques, Call taxi.

Submitted date 15 June 2013

Accepted Date: 20 June 2013

\section{INTRODUCTION}

A VANET or Vehicular Ad-Hoc Network is a formation of Vehicular Mobile ad-hoc network, to offer communications between nearby vehicles. In VANET, there is a regular requirement of the information about the current location, routes, specifically for data on the surrounding traffic, etc. This information can be combined together in a number of groups. This includes many dissimilar belongings mostly based on sensor data from other vehicles. Examples of VANET application are brake caution sent from preceding vehicle, track and collision caution, details about road condition and maintenance, detailed area weather prediction, forewarning of traffic jams, care to an accident behind the next turning, detail about an accident for the rescue team and many other things. Other examples are local updating of the vehicle navigation systems information or a subordinate that assists to chase a friend's vehicle. Another group is infotainment for passengers. For example, interactive games between vehicles close to each other. The kids will be in love with it. Next group is local information as next free parking space perhaps with a vehicle reservation system, detail about fuel prices and services offered through the subsequent service station or just tourist information about places of interest. A possible other group is vehicle maintenance. For example online help from a vehicle mechanic when your vehicle breaks down or just simply information about service. No further inter-vehicle communication system for data replacement between vehicles and among roadside and vehicles has been put into operation.

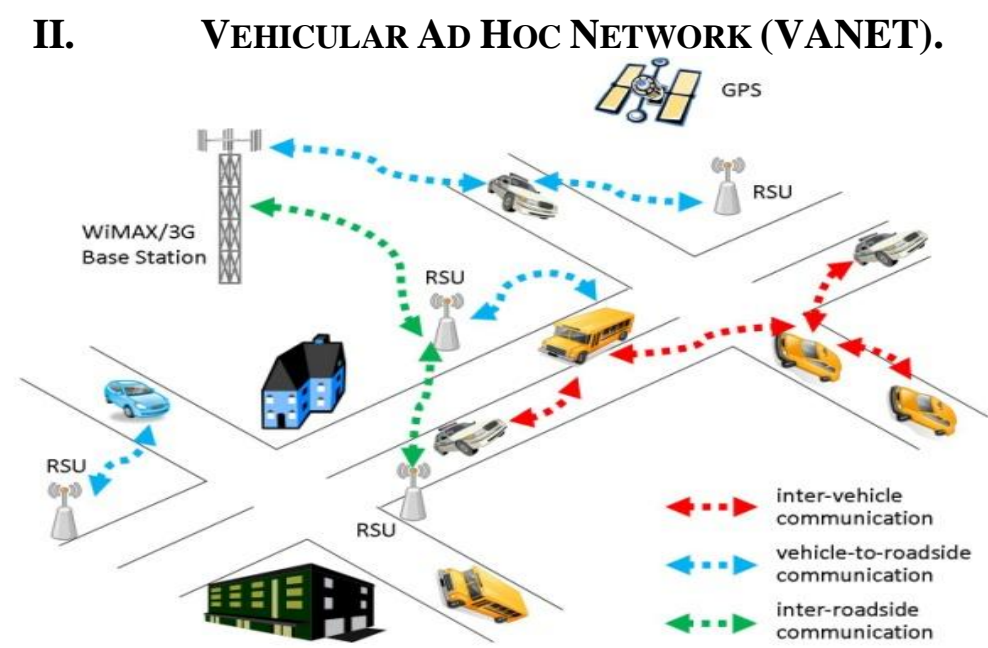

Fig 1.Vehicular Ad hoc NETwork (VANET).

Vehicular Ad-hoc Networks are expected to implement a variety of wireless technologies such as a type of WiFi called Dedicated Short Range Communications (DSRC). Other candidate wireless technologies are 
Satellite, WiMAX and Cellular. VANET (Vehicular Ad-hoc NETworks) can be viewed as component of the Intelligent Transportation Systems (ITS).

Vehicular Networks are a vision of the Intelligent Transportation Systems (ITS). Vehicles communicate with each other via Inter-Vehicle Communication (IVC) as well as with roadside base stations via Roadside-to-Vehicle Communication (RVC). The optimal goal is that vehicular networks will contribute to safer and more efficient roads in the future by providing timely information to drivers and concerned authorities. VANETs are characterized by their unique characteristics that distinguish them from MANET. These special characteristics can be summarized as follows:

- Higher mobility of nodes: VANET nodes are characterized by their high relative speed which makes VANET environment high dynamic.

- $\quad$ Predictable and restricted mobility patterns: Unlike the random mobility of MANET, VANET node movements are governed by restricted rules (traffic flow theory rules), which make them predictable at least on the short run.

- Rapid topology change: VANET nodes are characterized by their high speed. This leads to frequent network topology changes, which introduces high communication overhead for exchanging new topology information.

- $\quad$ No power constraints: Each vehicle is equipped with a battery that is used as an infinite power supply for all communications and computation tasks.

- Localization: Vehicles can use the Global Positioning System (GPS) to identify their locations with high accuracy.

- Abundant network nodes: Unlike MANETs that are characterized by a small network sizes, VANET networks can be very large due to high density of the vehicles.

Hard delay constraints: Safety messages are the main goal of VANETs. Therefore, safety messages should be given high priority and must be delivered on time.

\section{TRAFFIC MANAGEMENTS}

This type of applications is used to facilitate traffic flow, thus reducing traffic congestion, fuel consumption, and travel time. This type of applications is not as much of harsh on real-time constraints. This means that if the messages are delayed, there is no real threat to life i.e., no collision to occur. If the messages are delayed as contrasting to the safety messages where a real threat to the life may occur. The information provided by these applications mainly describes the status of the traffic in a certain areas like intersection or road constructions. In this kind of applications, vehicles cooperate to generate messages. Using inter-vehicle communications these messages are collected and sent, in a multi-hop manner to other vehicles in other geographic areas.

\section{Survey Of Routing Protocols}

This section provides a survey of routing protocols in vehicular ad hoc networks. The routing protocols are divided into two major categories,

- $\quad$ Topology-based routing

- $\quad$ Position-based routing.

The following section discusses the advantages and disadvantages of these routing protocols, discovers the inspiration following their trace the evolution and design of these routing protocols.

\section{Topology-based Routing Protocols}

These routing protocols use links' information that exists in the network to perform packet forwarding. They can further be categorized into

- $\quad$ Proactive (table-driven) routing

- $\quad$ Reactive (on-demand) routing.

\section{Proactive (table-driven):}

Proactive routing carries the distinct feature: the routing information such as the next forwarding hop is maintained regardless of communication requests in the background. Broadcast control packets constantly and flooded among nodes to maintain the paths or the link states between any pair of nodes even though some of paths are not at all used.

A Routing table is then constructed within a node such that each entry in the routing table indicates the next hop node toward a certain destination node. The merits of the proactive routing protocols are that there is no route discovery since route to the destination is maintained in the background and is always obtainable ahead lookup. Even though it's good property of providing low latency for real-time applications, the preservation of unused paths engages an important part of the obtainable bandwidth, particularly in highly mobile VANETs. 
Example of Proactive protocol Fisheye State Routing which is an efficient link state routing that maintains a topology map at each node and propagates link state updates with only immediate neighbors not the entire network. Furthermore, the link state information is broadcast in different frequencies for different entries depending on their hop distance to the current node. Entries that are further away are broadcast with lower frequency than ones that are closer. The reduction in broadcast overhead is traded for the imprecision in routing. However, the imprecision gets corrected as packets approach progressively closer to the destination.

\section{Reactive (On Demand):}

Reactive routing opens a route only when it is necessary for a node to communicate with one more nodes. It maintains only the routes that are at present in use, thus dropping the burden on the network. The reactive routings naturally have a route discovery phase where query packets are flooded into the network in search of a efficient path. The phase completes when a shortest path i.e., route is found.

S

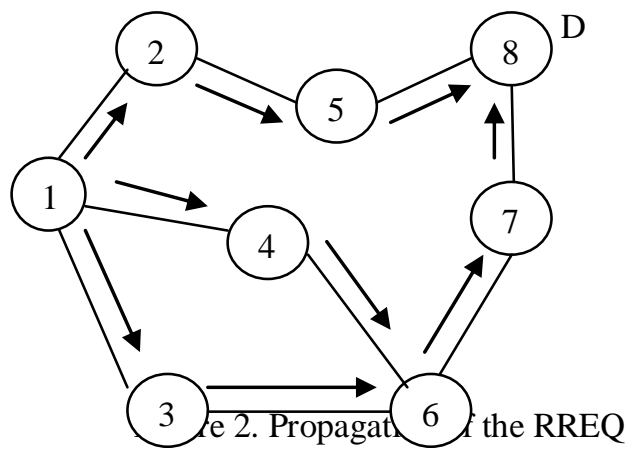

Example of Reactive Protocol is AODV - In Ad Hoc on Demand Distance Vector (AODV) (Perkins, 1999) routing, upon receipt of a broadcast query (RREQ), nodes record the address of the node sending the query in their routing table (Figure 3a).

This procedure of recording its previous hop is called backward learning. Upon arriving at the destination, a reply packet (RREP) is then sent through the complete path obtained from backward learning to the source (Figure 3b). At each step of the path, the node would record its previous hop, thus establishing the forward path from the source. The overflowing of query and throwing of reply creates a full duplex path.

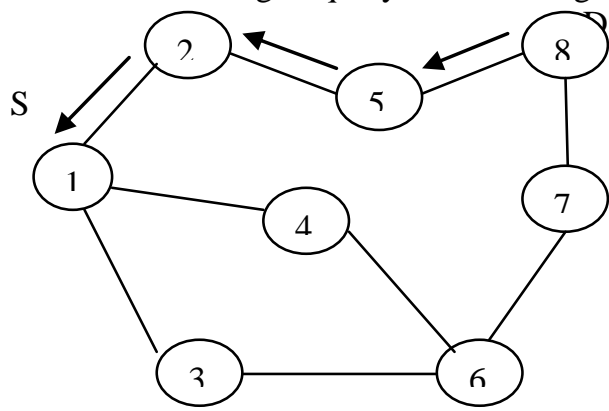

Figure 3. Path of the RREP to the source.

After the path has been established, it is maintained as long as the source uses it. A link failure will be reported recursively to the source and will in turn trigger another query-response procedure to find a new route.

\section{ArTi-Q In Existing ArtigenCe}

This is an existing system they used Artigence technique which is used for efficient call taxi management system. Artigence means that artificial intelligence, i.e., the capacity of a computer to perform operations analogous to learning and decision making by humans, as by an expert system. As per the name, can know that the Artigence system can perform tasks without the manpower. In the existing system Artigence is the technique which uses the algorithm called Arti-Q. Existing Arti-Q consists of two types of controlling component, they are,

- Arti-Q main

- $\quad$ Arti-Q proxy

The functionalities of the above two control units are described in the working strategy of the Arti-Q algorithm. The Existing algorithm uses the Queue data structure, which follows the strategy first in first out. The parallel 
processing will be carried out in the existing system at the time of finding the response for the respective request. In the existing system the Arti-Q algorithm works as follows,

- The data are established for the process from external proxy to internal proxy.

- At the first time, the Arti-Q main server will receive the requests. Request receiving in Arti-Q main will be discontinued after 0.5 second timer. The timer can be varying based on the nature of the application and requirement of the user.

- By the time of developing in the Arti-Q main server, the requests are received by the Arti-Q proxy.

- The demands i.e., requests are stocked up in the Queue data structure, in Arti-Q main and also in Arti-Q proxy.

- In Arti-Q main, the first request is processed and the response is found. Then Arti-Q main will analysis that the same request of the first process is repeated by other processes in the queue.

- As a result, the identical response will be sent to another process whose requests are same like the processed request.

- And then provided requests are removed from the queue.

The Arti - Q proxy will load the demands i.e., requests in the Arti-Q main for subsequent iteration.

\section{Artigence In Vanet}

Existing Artigence can be combined with VANET with the call taxi management systems, to manage the vehicle reservation more well-organized. For that externally one mobile is connected to the system. This mobile device is used for GSM communication and to increase the baud rate of the system. The existing Artigence system consists of the information about the vehicle and drivers. By using GPRS the system will recognize the vehicle's environmental location. As well the information will store in the vehicle information.

The customer who needs vehicle will send a message which should be in the syntax of "'taxi' space 'area name" to the toll free number. At the same time many number of users can send the requests to search out the vehicle. The Artigence is running to handle multiple requests in the server. The system finds the vehicle which is near to the user's location. The driver name, vehicle number and driver's mobile number will send to the user's mobile number. The vehicle reservation near the city will consists of some constraints that,

- The driver who was free at that time

- The driver who has low workload.

\section{Advantages of the Artigence}

The existing Artigence system which is used in the VANET for efficient call taxi management system consists of following advantages.

- Decreases waiting time of the requests in the queue.

- In the existing strategy, if any file or information needs to be transfer means we need web connection. But while using the Arti-Q we can transfer the information by using GSM communication.

- In the existing systems, the information can be hacked by the unauthorized users. But in the Arti-Q

system, because of GSM communication the information is secured while transferring it.

Current networking techniques are having many disadvantages. The main disadvantage is time consuming in the sense the response time of a particular request will be reduced in the Arti-Q system. But the response time of Arti -Q can be reduced by using Dual Arti-Q system.

Process distribution allows you to load cubes into separate sub processes that run parallel to the main process of the system. As a result of using process distribution, administrative observer can maximize the memory amount available for the cubes to be loaded.

\section{Parallel And Distributed Computing}

Distributed systems are groups of computers or networks, which contains the similar goal for their work. The similar system may be characterized both as "parallel" and "distributed"; the processors in a characteristic distributed system run simultaneously in parallel. Parallel computing may be seen as a particular tightly coupled form of distributed computing, and distributed computing may be seen as a loosely coupled form of parallel computing. Nevertheless, it is possible to roughly classify concurrent systems as "parallel" or "distributed" using the following criteria:

- In parallel computing, all processors may have access to a shared memory to exchange information between processors.

- In distributed computing, each processor has its own private memory. Data are exchanged by passing messages between the processors. 
(a)
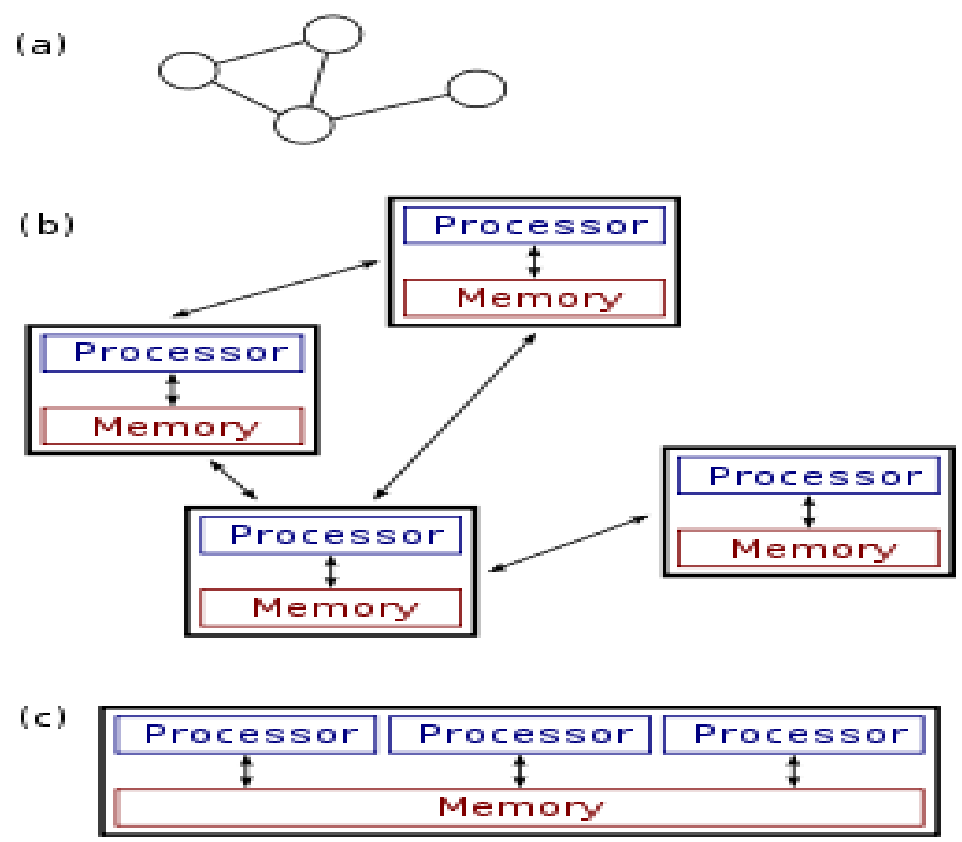

Figure 4. Parallel and distributed computing.

(a) - (b) A distributed system \& (c) Parallel system.

The figure on the right illustrates the difference between distributed and parallel systems. Figure (a) is a schematic view of a typical distributed system; typically, the system is characterized as a network topology in which each node is a computer and each line connecting the nodes is a communication link. Figure (b) shows the same distributed system in more detail: each computer has its own local memory, and data can be exchanged only by passing messages from one node to another by using the available communication links. Figure (c) shows a parallel system in which each processor has a direct access to a shared memory.

\section{DUAL (distributed) ARTI-Q SYSTEM}

In dual Arti-Q system is based on the existing Artigence system. In existing Artigence system the functionality is classified into two categories. The functionalities are divided and each functionalities are performed in a distributed manner. So the functionalities are,

- Scheduling

- Message Transmission

Scheduling

Scheduling is the process of deciding how to commit resources between a variety of possible tasks. Time can be specified (scheduling a flight to leave at 8:00) or floating as part of a sequence of events. Based on the definition of scheduling this phase is dealing with the following task alone.

- Receiving a request from the user or the customer in the syntax of "taxi" space "area name" to the toll free number.

- The system arranges the request in the FIFO queueing format.

- It finds the same requests if any. Based on that it rearranges the queuing structure.

- It finally finds the response i.e., the call taxi which was free at the time of response.

- And those details are stored in the scheduling Arti-Q server or scheduling Arti -Q system.

Message Transmission

Message transmission is the process of sending the response (call taxi contact number) based on the request ("taxi" space "area name") to the corresponding user or customer. So it consists of following functionalities,

- It refers scheduled and processed requests from the server.

- It checks the mobile number of the user who sent the requests

It finally sends the driver name and contact number of the taxi to the customer.

\section{Conclusion}

The concept of VANET and the characteristics of VANET is discussed in the first section. Routing and its types are explained in the second section. Routing is the process of finding the shortest path for message transmission. Section 3 and 4 are dealing with the existing concept of Artigence and how Artigence can be used 
to improve the efficiency of the calltaxi management system and it having the disadvantage related to the time. By using Dual Arti-Q system how the disadvantage of Artigence based call taxi management is avoided. So that the efficiency of Dual Arti-Q system is $40 \%$ improved when compared with existing Artigence based VANET in the application of Call taxi management system.

\section{REFERENCES}

[1] Cheng, P.-C., Weng, J.-T., Tung, L.-C., Lee, K. C., Gerla M., and Härri J. "GeoDTN+NAV: A Hybrid Geographic and DTN Routing with Navigation Assistance in Urban Vehicular Networks," (2008), Proceedings of the 1st International Symposium on Vehicular Computing Systems (ISVCS'08), Dublin, Irland, July 2008.

[2] Davis, J., Fagg, A. and Levine, B. "Wearable computers as packet transport mechanisms in highly-partitioned ad-hoc networks. in International Symposium on Wearable Computing,", 2001.

[3] Flury, R. and Wattenhofer, R. (2006), "MLS: an efficient location service for mobile ad hoc networks." In MobiHoc '06: Proceedings of the 7th ACM international symposium on Mobile ad hoc networking and computing, pages 226-237, New York, NY, USA, 2006.

[4] Forderer, D (2005). "Street-Topology Based Routing." Master's thesis, University of Mannheim, May 2005.

[6] Franz W., Eberhardt R., and Luckenbach T., "FleetNet - Internet on the Road," Proc. 8th World Congress on Intelligent Transportation Systems, Sydney, Australia, Oct. 2001.

[7] Giordano S., et al., "Position based routing algorithms for ad hoc networks: A taxonomy," in Ad Hoc Wireless Networking, X. Cheng, X. Huang and D.Z. Du (eds.), Kluwer, December 2003.

[8] Jaap, S., Bechler, M., Wolf, L. (2005), "Evaluation of Routing Protocols for Vehicular Ad Hoc Networks in City Traffic Scenarios," Proceedings of the 5th International Conference on Intelligent Transportation Systems (ITS) Telecommunications, June, 2005.

[9] Jerbi, M., Senouci, S.-M., Meraihi, R., and Ghamri-Doudane, Y. (2007), "An improved vehicular ad hoc routing protocol for city environments," Communications, 2007. ICC '07. IEEE International Conference, pp. 3972-3979, $24-28$ June 2007.

[10] Jetcheva, J.G., Hu, Y.-C., PalChaudhuri, S., Saha, A.K., Johnson, D.B. (2003), "Design and evaluation of a metropolitan area multitier wireless ad hoc network architecture," Mobile Computing Systems and Applications, 2003. Proceedings. Fifth IEEE Workshop on , vol., no., pp. 32-43, 9-10 Oct. 2003.

\section{Author's Profile}

S. Sujatha completed her undergraduate degree at Sri Sarada College for Women, Tirunelveli and has also completed post graduate level courses MCA and M.Phil at Bharathiar University, Coimbatore, India, and is currently pursuing her doctorate in Computer Science. Her area of interest is Mobile Agent Technology \& Networks. She has been participating continuously in research and development activities for the past ten years.

To her credit, she has presented and published technical papers in International Journals, at International Conferences and International Workshops organized by various international bodies like IEEE, WSEAS, and IEEE Explore. She has published book on Integrating SOA and Web Services and also contributed chapters on Personal Area Network and published articles \& working manuals in agent technology. The author is currently employed as Associate Professor at the Dr. G.R Damodaran College of Science, Coimbatore, India. She is an active member of various technical bodies like ECMA, Internet Society of Kolkata and Chennai and acts as a moderator in various international conferences and journals.

P.Sukanya completed her undergraduate degree at Don Bosco College, Panaji Goa and has also completed post graduate level courses MCA at Karunya University, Coimbatore, and is currently pursuing her Mphil in Computer Science at Dr. G.R Damodaran College of Science, Coimbatore, India. Her area of interest is Advance Networks. She has presented technical papers at National Conferences. 